\title{
Bacterial response to spatial gradients of algal-derived nutrients in a porous microplate
}

\author{
Hyungseok Kim (D ${ }^{1,2}$, Jeffrey A. Kimbrel $\mathbb{D}^{3}$, Christopher A. Vaiana ${ }^{1,4}$, Jessica R. Wollard $\mathbb{D}^{3}$, Xavier Mayali (iD) ${ }^{3 凶}$ and Cullen R. Buie ${ }^{1 凶}$ \\ (c) The Author(s) 2021
}

Photosynthetic microalgae are responsible for $50 \%$ of the global atmospheric $\mathrm{CO}_{2}$ fixation into organic matter and hold potential as a renewable bioenergy source. Their metabolic interactions with the surrounding microbial community (the algal microbiome) play critical roles in carbon cycling, but due to methodological limitations, it has been challenging to examine how community development is influenced by spatial proximity to their algal host. Here we introduce a copolymer-based porous microplate to coculture algae and bacteria, where metabolites are constantly exchanged between the microorganisms while maintaining physical separation. In the microplate, we found that the diatom Phaeodactylum tricornutum accumulated to cell abundances $\sim 20$ fold higher than under normal batch conditions due to constant replenishment of nutrients through the porous structure. We also demonstrate that algal-associated bacteria, both single isolates and complex communities, responded to inorganic nutrients away from their host as well as organic nutrients originating from the algae in a spatially predictable manner. These experimental findings coupled with a mathematical model suggest that host proximity and algal culture growth phase impact bacterial community development in a taxon-specific manner through organic and inorganic nutrient availability. Our novel system presents a useful tool to investigate universal metabolic interactions between microbes in aquatic ecosystems.

The ISME Journal (2022) 16:1036-1045; https://doi.org/10.1038/s41396-021-01147-x

\section{INTRODUCTION}

Metabolic interactions between microalgae and their associated bacteria, the latter sometimes referred to as the algal microbiome, have been recognized as an important contribution to carbon cycling in natural [1] and engineered [2] algal-dominated ecosystems. Heterotrophic bacteria consume up to $50 \%$ of the carbon fixed by algae [3], and this mineralization process often leads to an exchange of metabolites from bacteria to algae, providing a variety of micronutrients such as trace metals [4], vitamins [5-7], and phytohormones [8], which can be scarce in nature yet are essential for algal growth. Due to the potential influence of the algal microbiome on algal physiology, much effort has been made to identify algal-associated bacterial community structure and understand how it is related to algal diversity. In particular, it has been revealed that the algal-associated bacterial community can be highly conserved across time $[9,10]$, uniquely shaped by their algal host [11-13], and exhibits structural differences between algal-attached and free-living [13].

These studies indicate that the community-level bacterial response to algal hosts depends at least partially on physical proximity, including physical attachment, as well as the type of algal-excreted products. Maintaining physical proximity can significantly enhance the exchange rate of metabolites between microorganisms, which is especially important under an openocean environment where the nutrients are scarce [14] and marine aggregates can contribute to the majority of nutrient cycling [15]. Indeed, microscale interactions between algae and bacteria are commonly observed as direct cell-to-cell attachment $[11,12,16,17]$ or bacterial chemotaxis towards local gradients of algal exudates [18-21], with ongoing efforts to visualize these processes via micro or nano-scale chemical imaging [22, 23].

The predominant mechanism that explains how heterotrophic bacteria respond to algal-derived organic matter is the diffusive transport of metabolites. Metabolites diffusing around an alga create a spatial gradient of nutrients, and the region where the algal-derived organic nutrients are abundant is termed the phycosphere [1, 21, 24]. For an alga sized less than $100 \mu \mathrm{m}$, diffusion within its phycosphere plays a major role in transporting metabolites rather than fluid advection [1] or turbulence [25]. Moreover, diffusive transport is considered to be 50 times more efficient for encountering molecules than cellular swimming, especially at a low stirring number (defined as $I V / D ; l$ is intermolecular spacing, $v$ swimming speed, and $D$ diffusion coefficient) $[14,26]$. Therefore, by maintaining spatial proximity to the algal cell inside the phycosphere, bacteria can best exploit algal-derived organic matter through diffusive effects.

In order to assess how microorganisms respond to the diffusion of metabolites such as algal exudates, in vivo co-culture methods have been developed. For example, a dual-chamber system separated by a porous membrane [20, 27-29] has enabled further downstream biological analyses, such as plate reader-compatible in situ growth measurements [28], metabolomics [27], or

\footnotetext{
${ }^{1}$ Department of Mechanical Engineering, Massachusetts Institute of Technology, Cambridge, MA, USA. ${ }^{2}$ Institute for Data, Systems, and Society, Massachusetts Institute of

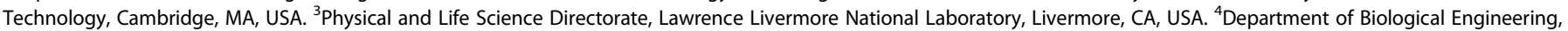
Massachusetts Institute of Technology, Cambridge, MA, USA. ${ }^{凶}$ email: mayali1@llnl.gov; crb@mit.edu
} 
microscopy to track cellular motility [20] and biofilm formation [29]. Microscopy has indeed provided valuable information on how bacteria can temporally respond to the presence of algae via chemotaxis $[19,20]$. Nonetheless, existing techniques have not yet enabled the co-cultivation of microalgae with multiple bacterial species, especially for time periods long enough to detect community-level dynamics (days to weeks).

In this paper, we present a porous microplate targeted to the study of in vivo interactions between algae and free-living bacteria. We develop a protocol to culture the microorganisms for up to several weeks in the porous microplate, overcoming previous limitations mentioned above. We employ the copolymer poly(2-hydroxethyl methacrylate-co-ethylene glycol dimethacrylate) (HEMA - EDMA), previously shown to be an effective building copolymer to enable cell-to-cell communication ranging from microbial [30] to mammalian cells [31]. Using this approach, we set our central goal to understand how metabolite diffusion plays a role in the interaction between algal cells and their associated bacteria. We explore how free-living bacterial populations respond to algal exudate diffusion by altering its input to bacterial communities through changing the physical proximity between the source (the algae) and the sink (the bacteria). Specifically, by taking advantage of our incubation system (Fig. 1a), we hypothesize that inorganic nutrients from the medium are converted into dissolved organic carbon (DOC) through algal photosynthetic activity in vivo and that the conversion creates a spatial gradient of DOC and inorganic nutrients across the culture wells, the response to which can be detected by changes in bacterial abundance and community structure.

To test our hypotheses, we designed three consecutive experiments with algae and free-living bacteria incubated in the porous microplate system. We first examined the model alga Phaeodactylum tricornutum grown in porous microplate wells that are spatially arranged to generate different nutritional availability and quantify growth (Fig. 1b). Next, we co-cultured $P$. tricornutum with two commonly algal-associated bacterial strains (of the genera Algoriphagus and Marinobacter), where the bacteria were incubated at different distances from the alga (Fig. 1c). These bacteria were previously isolated from $P$. tricornutum mesocosms
$[11,16]$ and have been shown to affect algal growth: Marinobacter increased the cell size, abundance and lipid content of $P$. tricornutum, and Algoriphagus incorporated more of the $P$. tricornutum exudates than Marinobacter [32]. Although the strains are phycosphere-associated, they rarely or do not attach to $P$. tricornutum (Supplementary Fig. S1), thereby allowing us to use them as free-living model organisms targeted to study certain aspects of the phycosphere interaction. Finally, we incubated $P$. tricornutum in the porous microplate with mixed bacterial communities to observe how community structure responds to physical distance away from the algal host (Fig. 1d).

\section{MATERIALS AND METHODS}

Acrylic and polydimethylsiloxane (PDMS) molds preparation The incubating device for the porous microplate was designed using a CAD software (Solidworks, Dassault Systèmes) and the exported drawing files were used to laser cut 1/4" and 1/8" acrylic sheet (Universal Laser Systems; Supplementary Fig. S2). After washing the cut acrylic parts with deionized water, they were attached by acrylic (Weld-On) and epoxy ( $3 \mathrm{M})$ adhesives that were followed by a curing process for $\sim 18 \mathrm{~h}$. Polydimethylsiloxane (PDMS) (Sylgard 184, Dow Corning) was cast onto the acrylic mold and cured at $80^{\circ} \mathrm{C}$ for at least $3 \mathrm{~h}$. The PDMS mold was carefully detached from the acrylic surface by dispensing isopropyl alcohol (VWR) into the area between the PDMS and the acrylic molds (Fig. 2a).

\section{Porous microplate preparation}

Synthesis of copolymer HEMA-EDMA was based on previously described protocols [30, 31] and details are given as follows. Prepolymer solution HEMA - EDMA was prepared by mixing 2-hydroxyethyl methacrylate (HEMA; monomer, 24 wt.\%, Sigma-Aldrich), ethylene glycol dimethacrylate (EDMA; crosslinker, 16 wt.\%, Sigma-Aldrich), 1-decanol (porogen, 12 wt.\%, Sigma-Aldrich), cyclohexanol (porogen, 48 wt.\%, Sigma-Aldrich) and 2,2dimethoxy-2-phenylacetophenone (DMPAP; photoinitiator, 1 wt.\%). The solution was stored at room temperature without light exposure until further use. Glass slides $\left(75 \times 50 \mathrm{~mm}^{2}\right.$, VWR) were chemically cleaned by sequentially soaking in $1 \mathrm{M}$ hydrochloric acid and $1 \mathrm{M}$ sodium hydroxide for one hour, followed by rinsing with deionized water and air drying. The prepolymer solution was cast onto the PDMS mold and a glass slide was placed on the mold. The solution was then polymerized under ultraviolet light with a wavelength $365 \mathrm{~nm}$ for 15 min by using a commercial UV lamp a

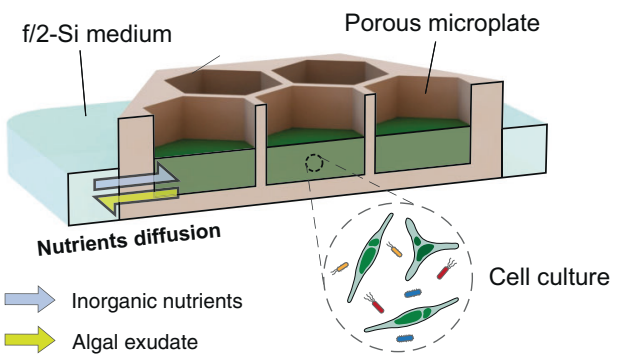

C

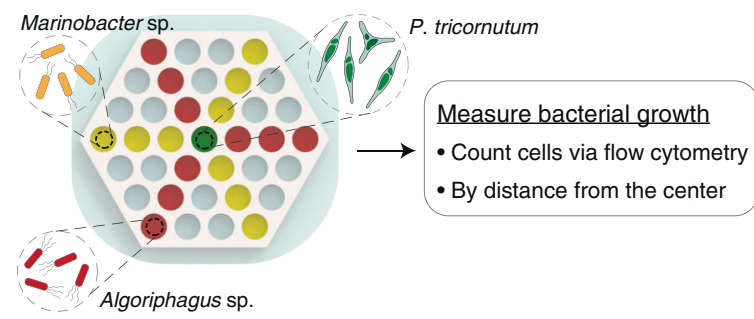

b Experiment 1. Axenic $P$. tricornutum growth

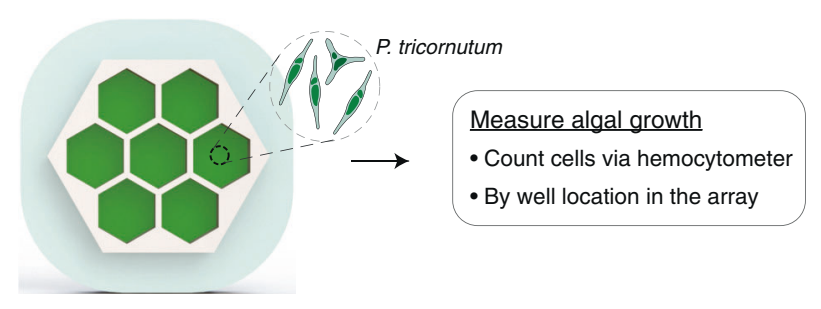

d Experiment 3. Algal-bacterial community analysis

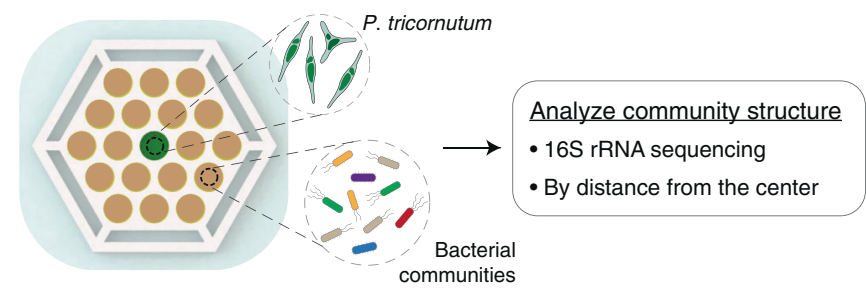

Fig. 1 Porous microplate for algal-bacterial interaction. a Cross-sectional schematic illustrating cell culture in a porous microplate. b-d Three experimental designs showing a top-view schematic of microplate wells to measure the growth of (b) P. tricornutum, (c) bacterial isolates, and (d) to analyze community structure of algal-associated bacteria. To avoid cross-contamination between adjacent wells in the coculture microplate, each well was designed with a rounded shape as the polygonal shape generates a sharp liquid meniscus near the top of the microplate [63]. 
a (1)

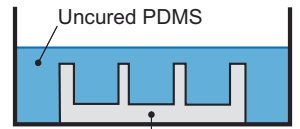

Acrylic mold

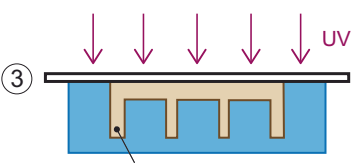

Prepolymer solution
(2)

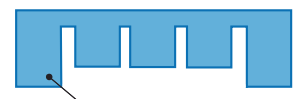

PDMS mold

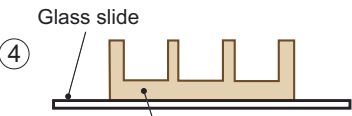

Porous microplate
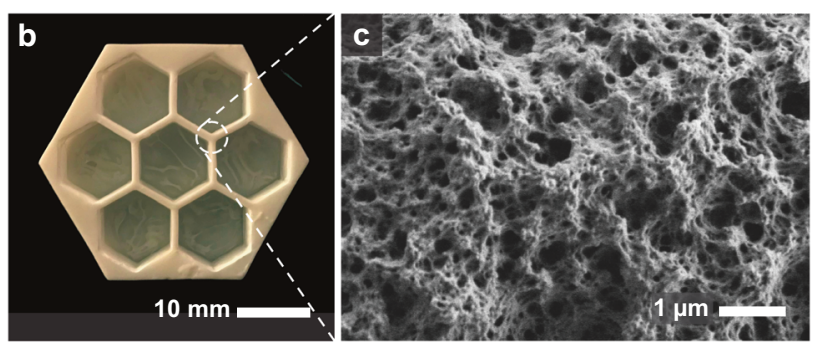

Fig. 2 Synthesis and characterization of porous microplate. a Procedure to build a porous microplate using polydimethylsiloxane (PDMS) and acrylic molds. b Image of the microplate with an array of culture wells (wall thickness: $0.9 \mathrm{~mm}$ ). c Scanning electron microscopy image of nanoporous copolymer HEMA-EDMA.

(VWR). The photopolymerized device was detached from the PDMS mold and stored in a jar containing methanol (VWR) until further use (Fig. 2a). The jar was refilled with new methanol twice in order to remove the remaining porogen and uncrosslinked monomers from the hydrogel.

Upon each incubation experiment with the porous microplate, each device was decontaminated by replacing the solvent with $70 \%$ alcohol (VWR) and storing it for $24 \mathrm{~h}$. They were immersed in a pre-autoclaved jar for two weeks with $f / 2$ medium with omitted silicate, where the jar was refilled once with a new sterile medium to adjust its $\mathrm{pH}$ for the algal culture and remove any solvent remaining in the hydrogel. Before inoculating microbial cells, each microplate was taken out from the jar and the media remaining on the top surface was removed by absorbing it with a pre-sterilized wipe to minimize the chance for cross-contamination between wells (Fig. 2b).

\section{Scanning electron microscopy}

Photopolymerized HEMA - EDMA was removed from methanol and dried in air for at least one week to evaporate the excess solvent. A $\sim 5 \times 5 \mathrm{~mm}^{2}$ specimen was collected from the dried copolymer and attached to a pin stub. The stub was loaded on a scanning electron microscope (SEM; MERLIN, Carl Zeiss), and the specimen was characterized with imaging software (SmartSEM, Carl Zeiss) with 16,270X magnification and an operating voltage of $1 \mathrm{kV}$. The SEM imaging was performed at the Electron Microscopy Facility in the MIT Materials Research Science and Engineering Centers (MRSEC; Fig. 2c).

\section{Strains and culturing conditions}

Axenic P. tricornutum CCMP 2561 was acquired from the National Center for Marine Algae and Microbiota (NCMA) and shown to be axenic via epifluorescence microscopy and sequencing of the $16 \mathrm{~S}$ rRNA gene [11]. $P$. tricornutum was maintained in $\mathrm{f} / 2$ medium with $20 \mathrm{~g} \mathrm{~L}^{-1}$ commercially available sea salts (Instant Ocean, Blacksburg) and with omitted silicate, which we will refer to as $\mathrm{f} / 2-\mathrm{Si}[11,16]$. Batch cultures were grown at $20^{\circ} \mathrm{C}$ with a $12 \mathrm{~h}$ light $/ 12 \mathrm{~h}$ dark diurnal cycle and a light intensity of $200 \mu \mathrm{mol}$ photons $\mathrm{m}^{-2} \mathrm{~s}^{-1}$ (Exlenvce). Every 2-3 weeks, axenic cultures were monitored for bacterial contamination by streaking culture samples on marine broth agar [33], that tests for contamination by bacteria that can grow on agar media and is not definitive. Every 6-12 months, every axenic and bacterial co-culture of $P$. tricornutum was inspected for the absence/ presence of bacteria by staining the cellular DNA with $0.1 \% \mathrm{v} / \mathrm{v}$ SYTO BC Green Fluorescent Acid Stain (Thermofisher, Supplementary Fig. S1).

Bacterial community samples (referred to as "phycosphere enrichments") were obtained from mesocosms of $P$. tricornutum and maintained as previously described $[11,16]$. Briefly, an outdoor $P$. tricornutum mesocosm sample in natural seawater was collected in Corpus Christi, TX and filtered with $0.6-1 \mu \mathrm{m}$ pores to remove larger algal cells. The bacterial filtrates were inoculated to an axenic algal culture, maintained in $\mathrm{f} / 2$-Si media for $\sim 3$ months, and washed with a sterile medium to enrich for phycosphere-associated bacteria. These enriched communities were subsequently co-cultured with $P$. tricornutum in $\mathrm{f} / 2$-Si media for $\sim 4$ years prior to the start of the experiments.

Two bacterial strains, Marinobacter sp. 3-2 and Algoriphagus sp. ARW1R1, were isolated from the phycosphere enrichment samples (Supplementary Table S1). The isolates were either maintained by growing on marine broth agar plates at $30^{\circ} \mathrm{C}$ or by co-culturing with $P$. tricornutum through inoculation of a single colony into the axenic culture.

\section{P. tricornutum culture in porous microplate}

Three baseline experiments were designed to study how the alga $P$. tricornutum interacts with its associated bacteria in the porous microplate (Fig. 1). For experiments assessing the algal growth in the microplate, axenic $P$. tricornutum was acclimated to a copolymer environment in advance by inoculating a stationary phase-culture to a separate microplate. After acclimation for 4 days, the culture was diluted to $\sim 1 \times 10^{6}$ cells ml $^{-1}$ and transferred to the experimental microplate. Three replicated microplates were placed in a single transparent covered container $(128 \times 85 \times$ $10 \mathrm{~mm}^{3}, V W R$ ) which was filled with $\sim 25 \mathrm{ml} / 2$-Si medium to keep the microplate hydrated throughout the incubation period of 20 days with an initial culture volume of $75 \mu \mathrm{l}$ (Fig. 1a). The procedures were conducted under a biosafety cabinet to prevent any biological contamination. The cells were incubated under the same conditions as described above for the batch cultures (temperature, light intensity, diurnal cycle).

Growth of $P$. tricornutum was measured by counting cells using a hemocytometer (Electron Microscopy Sciences) or flow cytometry (described later). Specific growth rates were calculated from the natural log of the cell densities in triplicate sampled during an exponential growth phase (day 3 for the batch culture, day 5 for the porous microplate system; Fig. 3a).

\section{Algal-bacterial isolates experiment}

Marinobacter sp. 3-2 and Algoriphagus sp. ARW1R1 were prepared by inoculating a single colony into marine broth and growing overnight $\left(30^{\circ}\right.$ C, 150 r.p.m.). The cells were washed with $\mathrm{f} / 2$-Si twice by centrifuging at $2258 \mathrm{rcf}$ for $4 \mathrm{~min}$ and diluted to a density of $\sim 4 \times 10^{6}$ cells ml $^{-1}$

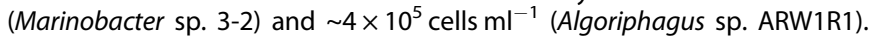
The cell densities were initially set to resemble in situ conditions with $P$. tricornutum-associated bacterial communities where Marinobacter displayed relative abundances several fold higher than Algoriphagus (Supplementary Note S1) [16]. The diluted bacterial cultures were transferred to the surrounding wells of the experimental microplate with pre-acclimated $P$. tricornutum with a density of $\sim 1 \times 10^{7}$ cells ml $^{-1}$ in the center well, as described above (Fig. 1c).

Every 2-3 days, $5 \mu \mathrm{l}$ samples were collected from wells and transferred to a 96-well plate (Corning). Before collecting the samples, the culture was thoroughly mixed using a micropipette to avoid sampling bias resulting from any spatial heterogeneity. Cells were then diluted 35 times with $1 \mathrm{x}$ phosphate-buffered saline solution (PBS) (Mallinckrodt), followed by the addition of $16 \%$ formaldehyde (Thermo Scientific) to a final concentration of $2 \%$ adjusting to a final volume of $200 \mu \mathrm{l}$. Fixed samples were stored at $4{ }^{\circ} \mathrm{C}$ for no more than 4 weeks. After collecting the cell samples for 20 days, the remaining cultures were taken out from the device and were streaked on marine broth agar plates to check if any of the culture wells had been crosscontaminated, based on different colonies morphologies (Supplementary Fig. S3). We detected cross-contamination in 5 out of 108 bacterial samples, and they were excluded from the analysis. No less than three replicates were retained after excluding the cross-contaminated samples.

\section{Flow cytometry}

Fluorescent counting beads for flow cytometry (Alignflow ${ }^{\mathrm{TM}}$, Thermofisher) were diluted 20 times with PBS and $50 \mu \mathrm{l}$ was added to each well of the 96well plate for calibration. SYBR Green I nucleic acid stain (Thermofisher) was added to the samples with a final concentration of $0.1 \% \mathrm{v} / \mathrm{v}$, and allowed to sit at room temperature at least for $30 \mathrm{~min}$ without light exposure. 
a

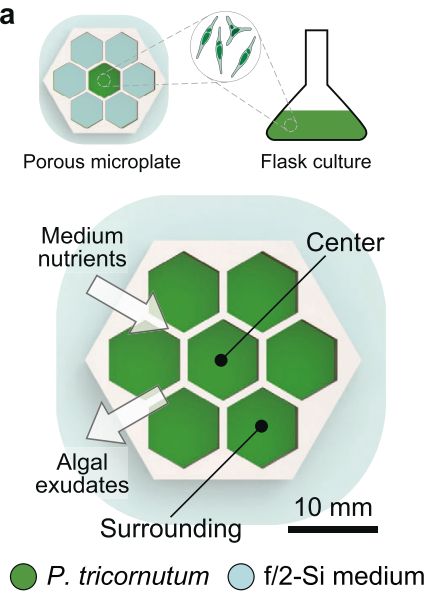

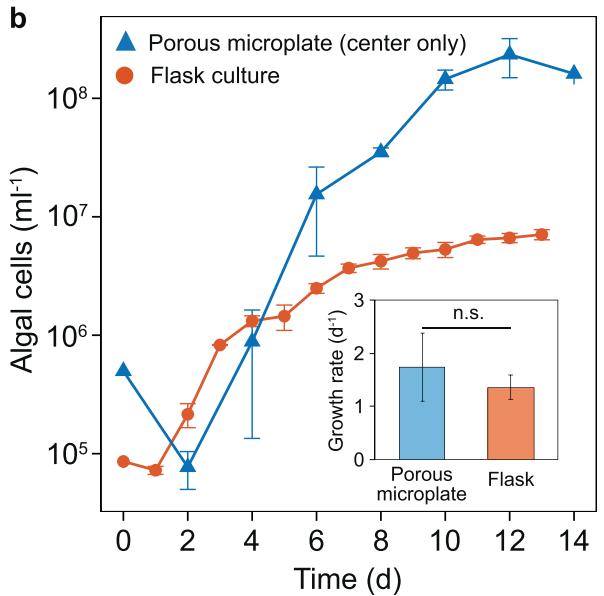

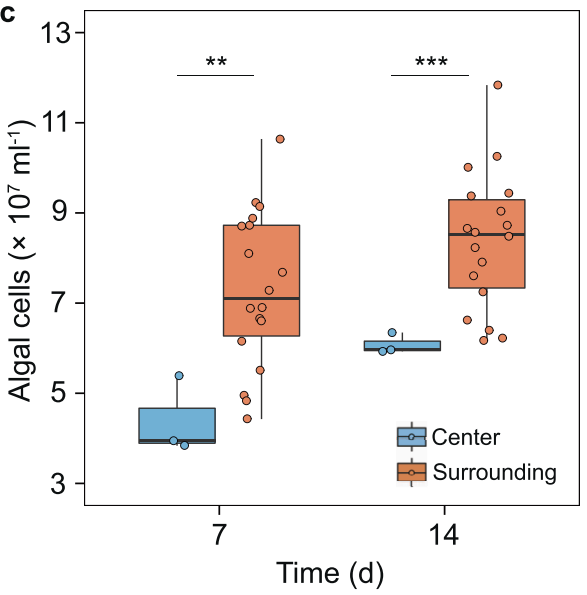

Fig. 3 Cultivation of $P$. tricornutum in the porous microplate. a Schematic of a microplate for algal cultivation. $\mathbf{b}$ Growth curve and maximal growth rate (inset) comparing the porous microplate with flask culture. Error bars, standard deviation of triplicates. c Cell abundance at center $(n=3)$ and surrounding $(n=18)$ wells after incubation. Asterisks denote statistical differences with following levels (two-tailed $t$-test): ${ }^{* * *} P<$ $0.001,{ }^{* *} P<0.01,{ }^{*} P<0.05$ and n.s. not significant.

Flow cytometry analysis was conducted on a BD FACS Canto II HTS to quantify the number of algal and bacterial cells with parameter setting as follows: (voltage) $\mathrm{FSC}=580, \mathrm{SSC}=370, \mathrm{GFP}=400, \mathrm{PE}=330, \mathrm{PerCP}=647$, PE-Cy7 $=677$, Alexa Fluor $680=290$, APC-Cy $7=410$, Pacific Blue $=440$, AmCyan $=539$. Populations were plotted with GFP-A and APC-Cy7-A intensities, allowing a clear distinction between counting beads, stained algal, and bacterial cells. The data were exported to.csv files using FlowJo (BD) and converted into cell density data using MATLAB (Mathworks).

\section{Bacterial community experiment}

To examine bacterial community structure changes induced by the presence of the diatom, $\sim 1 \times 10^{6} \mathrm{P}$. tricornutum cells $\mathrm{ml}^{-1}$ were placed in the center well and the results were compared to control incubations where sterile media was placed in the center (Fig. 1d). Phycosphere enrichment samples $[11,16]$ (with the algal cells removed with a $0.8 \mu \mathrm{m}$ filter) were used as a bacterial community inoculant where $100 \mu \mathrm{l}$ samples were placed in the surrounding wells in the array. Layer 1 and Layer 2 refer to bacterial wells with a distance from the centre well of $8 \mathrm{~mm}$ and $16 \mathrm{~mm}$, respectively. On the outmost well, $100 \mu \mathrm{l}$ sterile $\mathrm{f} / 2$-Si medium was inoculated as a blank control for $16 \mathrm{~S}$ rRNA community analysis.

The devices were incubated in a container $\left(\right.$ GasPak $^{\mathrm{TM}}$ EZ container systems, BD) which was filled with $\mathrm{f} / 2$-Si medium. All preparation steps were performed in a laminar flow hood to prevent any bacterial contamination. The container was incubated under the same condition as described above. After 7 days, each well sample was collected and filtered into 96 -well filter plate ( $0.2 \mu \mathrm{m}$ pore size, Pall AcroPrep) to remove liquid, and was stored at $-20^{\circ} \mathrm{C}$ until further analysis.

\section{$16 \mathrm{~S}$ ribosomal RNA gene amplification and sequencing}

Twenty microliters of sterile DNA-free water were added to each well of the filter plates, and cells were lysed to release the DNA with heat $\left(95^{\circ} \mathrm{C}\right.$ for $10 \mathrm{~min}$ ). The $16 \mathrm{~S}$ rRNA gene was directly amplified without cleanup, using primers targeting the $v 4$ region and modified to include lllumina platform adaptor sequence on the $5^{\prime}$ ends. The PCR contained $10 \mu \mathrm{l}$ of 5 Prime MasterMix, $1 \mu \mathrm{l}$ of $10 \mu \mathrm{M}$ of forward $16 \mathrm{~S}$ primer (5' - TCGTCGGCAGCGTC AGATGTGTATAAGAGACAG[GTGYCAGCMGCCGCGGTAA] - 3') [34], $1 \mu \mathrm{l}$ of reverse $16 \mathrm{~S}$ primer $\left(5^{\prime}\right.$ - GTCTCGTGGGCTCGGAGATGTGTATAAGAGACAG [GGACTACNVGGGTWTCTAAT] -3') [35], and $5 \mu$ l of DNA template (actual primer sequences highlighted with brackets). Cycling conditions were as follows: denaturation at $94^{\circ} \mathrm{C}$ for $3 \mathrm{~min}$, followed by 30 cycles of denaturation at $94^{\circ} \mathrm{C}$ for $45 \mathrm{~s}$, annealing at $51{ }^{\circ} \mathrm{C}$ for $30 \mathrm{~s}$ and extension at $72^{\circ} \mathrm{C}$ for $1.0 \mathrm{~min}$. The final extension was conducted at $72^{\circ} \mathrm{C}$ for $10 \mathrm{~min}$ and the samples were held at $4{ }^{\circ} \mathrm{C}$. The second round of amplification added Illumina Dual Nextera XT indexes and sequencing adaptors [36]. Each library was quantified with a Qubit broad range dsDNA assay and equimolar amounts of each library were pooled. The size and concentration of the final pool were verified by a D5000 High Sensitivity assay on the Agilent Tapestation. Six pM of the pooled libraries were combined with $15 \%$ phiX and paired-end sequenced on an Illumina MiSeq for 500 cycles.

Paired-end MiSeq reads were filtered to remove read pairs that contained Illumina adapter or barcode kmers with bbduk v38.22 using options $k=31$ and hdist $=1$ [37]. Read trimming and quality filtering was done with DADA2 v1.12.1 using options trimLeft $=c(4,14)$, truncLen $=c$ $(200,150), \operatorname{maxN}=0, \max E E=c(2,2)$, truncQ $=2$ [38]. Read pairs remaining after filtering were further processed into amplicon sequence variants (ASVs) retaining ASVs of length 274 nucleotides with at least 2 reads in at least 2 samples. The sequences were then aligned with MUSCLE v3.8.1551 [39] and clustered into an approximately-maximum-likelihood tree with Fasttree v2.1.10 [40]. Taxonomy was assigned using IDTAXA [41] against the Silva version $132 \mathrm{SSU}$ database [42]. ASVs were analyzed using the $\mathrm{R}$ package phyloseq v1.30.0 [43]. Samples were quality-filtered by removing taxa not found at least 10 times in at least 3 samples and normalized by Cumulative Sum Scaling (CSS) method using the $\mathrm{R}$ package metagenomeSeq v1.28.2 [44]. In brief, a mean of 95,770 (from 11,535 to 241,656 ) and 1477 (from 61 to 5183) read pairs were sequenced per sample of bacterial wells and of quality-filtered negative controls, respectively (Supplementary Table S2). Quality-filtered negative samples have been selected from 18 total negative samples based on their read pairs of less than 10,000 . The other five negative control samples with reading pairs of more than 10,000, were dropped from the analysis as they suggested that cross-contamination had occurred from the adjacent bacterial communities for these samples. Another 36 bacterial cultures, located in between layers 1 and 2, were not assigned with a layer number and excluded from the statistical analysis. R package phyloseq $v 1.30 .0$ was used to perform principal coordinate analysis ( $\mathrm{PCOA}$ ), vegan v2.5.6 to perform permutational multivariate analysis of variance (PERMANOVA) [45] based on weighted Unifrac [46], and R function (aov) was used to perform one-way and two-way analysis of variance (ANOVA) tests using packages tidyverse v1.3.0 [47], vegan v2.5.6 [48].

\section{RESULTS}

\section{P. tricornutum accumulates in a porous microplate}

HEMA-EDMA is a nanoporous copolymer and can easily dry out in standard laboratory conditions (Fig. 2b, c). To circumvent the dehydrating issue, the porous microplates were immersed in $\mathrm{f} / 2-\mathrm{Si}$ medium in a single-well plate with a lid where $~ 95 \%$ of the initial $100 \mu \mathrm{l}$ volume was retained over two weeks (Supplementary Table S3). We measured cell abundances of $P$. tricornutum incubated in the center well of the microplate and compared them with conventional batch cultures (Fig. 3a). Unexpectedly, $P$. tricornutum in the porous microplate reached a density of $(2.3 \pm$ $0.8) \times 10^{8}$ cells $\mathrm{ml}^{-1}$, which was $\sim 20$ fold higher than those under a batch condition (Fig. 3b). However, the two culture methods did 
not show a difference in the growth rate, suggesting the copolymer did not impact $P$. tricornutum physiology once it was acclimated to the environment ( $P=0.413$, Fig. $3 \mathrm{~b}$ inset). A rapid drop in cell densities was also observed at the beginning of the microplate incubation (day $0-2$, Fig. $3 b$ ), presumably due to the lower pKa of the monomer hydroxyethyl methacrylate (HEMA) than the $\mathrm{pH}$ of $\mathrm{f} / 2-\mathrm{Si}$ medium [49], which likely impacted cell physiology initially.

We hypothesized that the higher carrying capacity in the microplate might be caused by the $\mathrm{f} / 2-\mathrm{Si}$ medium nutrients diffusing into the well as the algae grew, since nutrients are constantly consumed and a concentration gradient of nutrients would be created across the porous HEMA-EDMA [30]. We believe nutrient transport by fluid flow across the porous structure can be neglected, as the rate is small compared to the gradient-induced diffusion (Supplementary Note S2). We also believe vapor transport from surrounding culture wells can be neglected, as we did not observe any notable replenishment of the well volumes, and the nutrients (nitrate, phosphate) have low volatility. To test our hypothesis on the nutrient diffusion by the concentration gradient, we designed a porous microplate of a well array, one center and six outer wells, so that nutrients outside the microplate can supplement the outer wells faster than the inner wells (Fig. 3a). Indeed, algal populations cultured in the outer wells reached higher concentrations than cultures in the center $(P<0.01$, Fig. $3 c)$, but those at both locations still exhibited higher abundances than standard batch cultures. Growth rates in the surrounding wells were also statistically higher, $0.25 \pm 0.04 \mathrm{~d}^{-1}$, compared to the center wells, $0.18 \pm 0.03 \mathrm{~d}^{-1}(P<0.05$, two-tailed $t$-test). One further experiment confirmed that the inorganic nutrients in the outer reservoir diffused into the culture wells; we compared $P$. tricornutum abundances in unaltered medium with post-experiment reservoir medium, testing if the spent medium had lower nutrient concentrations. As expected, $P$. tricornutum grew slower and to lower concentrations in this spent medium compared to fresh medium, consistent with the hypothesis of inorganic nutrient diffusion from the reservoir into the wells (Supplementary Fig. S4).

\section{Predictive modelling of nutrient concentrations around an alga and in the porous microplate}

After observing $P$. tricornutum drawdown of inorganic nutrients in the wells of the porous microplate, we further designed it to build a testable analogous model to the microscale processes that occur at the single cell level in the algal phycosphere [24]. At the scale of a single alga, its photosynthetic activity creates two contrasting concentration gradients governed by the laws of diffusion: an increase of algal exudates and a decrease of inorganic nutrients towards the cell. Similarly, the location of the porous microplate wells at different distances from the algal culture can artificially create distinct zones comprised of different concentrations of both algal-derived metabolites and medium inorganic nutrients, although it does not specifically test algal-attached bacteria (Fig. 4a).

To determine how the porous microplate system can mimic the phycosphere environment from the point of view of algalassociated bacteria, we constructed a numerical model comparing spatiotemporal profiles of nutrients and exudates at these two different scales. For the phycosphere model, we tested four taxonomically diverse phytoplankton species, using published data from laboratory cultures [50]. This allowed us to infer spatial concentrations of dissolved organic carbon (DOC) and nitrate, as proxies of algal exudates and inorganic nutrients respectively, to directly compare to the modeled profiles in the porous microplate system with $P$. tricornutum (Supplementary Note S3). Regardless of growth phase, we found algal released compounds decrease in concentration by 3-7 fold with increasing distance from the cell surface, supporting the theoretical validity of the phycosphere $[1,24]$ (Fig. 4b, Supplementary Fig. S5). However, inorganic nutrients are rapidly consumed by the cells while they grow $[50,51]$, and the concentration of nitrate becomes low as observed in the numerical model when the culture reaches stationary phase (Fig. 4c, Supplementary Fig. S5). Similar results were obtained in the simulated porous microplate system, with a $\sim 5$ fold decrease in DOC concentration with increasing distance from the center alga-located well as well as the depletion of nitrate in the center well within 6 days (Fig. 4b, c).

\section{Bacteria respond to gradients of $\boldsymbol{P}$. tricornutum exudate and} medium nutrients

Following the experimental and numerical results on nutrient availability around the algal host, we next explored how algalassociated bacteria grow and respond to the diffusion of algal and medium nutrients in the porous microplate. In an array of microplate wells, $P$. tricornutum and two bacterial strains, Marinobacter sp. 3-2 and Algoriphagus sp. ARW1R1, were a
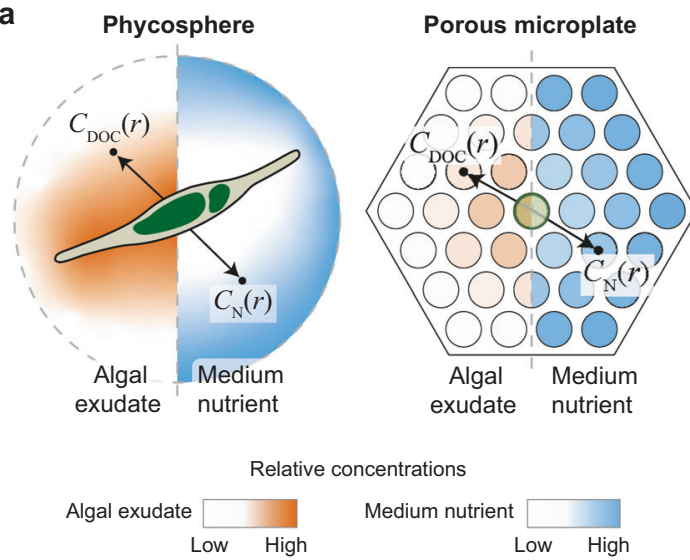

b
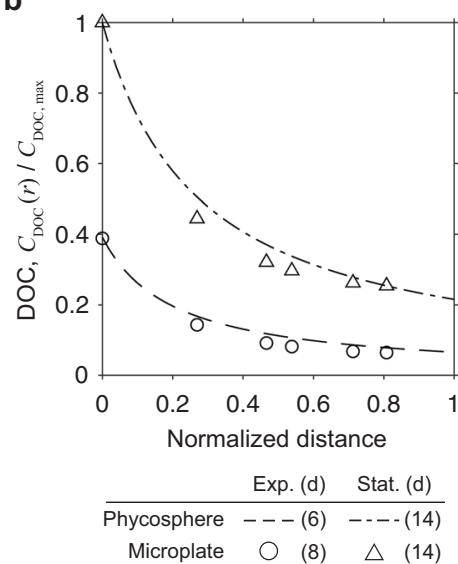

c

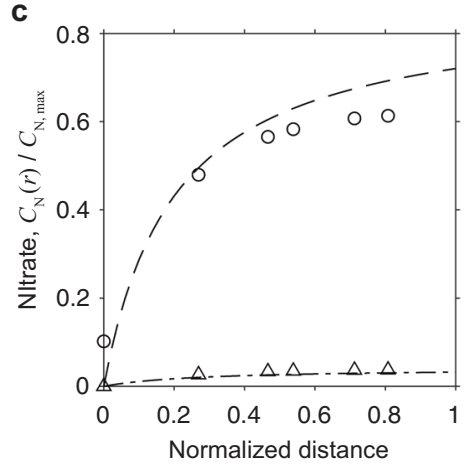

$$
\begin{array}{ccc} 
& \text { Exp. (d) } & \text { Stat. (d) } \\
\hline \text { Phycosphere } & ---(6) & ----(14) \\
\text { Microplate } & 0 & (5)
\end{array}
$$

Fig. 4 Numerical estimation of nutrient concentrations. a Schematic of two contrasting concentration gradients of algal dissolved organic carbon (DOC) and medium nutrients around a cell and in the porous microplate with $P$. tricornutum at the center and without bacteria. Numerical simulation of (b) DOC and (c) nitrate concentrations comparing the phycosphere (Synechococcus bacillaris) and the porous microplate at exponential (exp.) and stationary (stat.) growth phases. Nutrient concentrations and distances were normalized by their maximal values (Supplementary Note S3). Parameters for estimating phycosphere concentrations were based on data previously collected in a separate study [50]. 
a

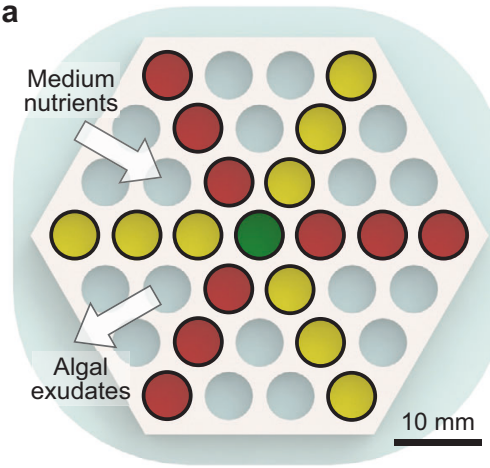

P. tricornutum $(\mathrm{Pt}) \bigcirc \mathrm{f} / 2-\mathrm{Si}$ medium

Marinobacter sp. Algoriphagus sp. b

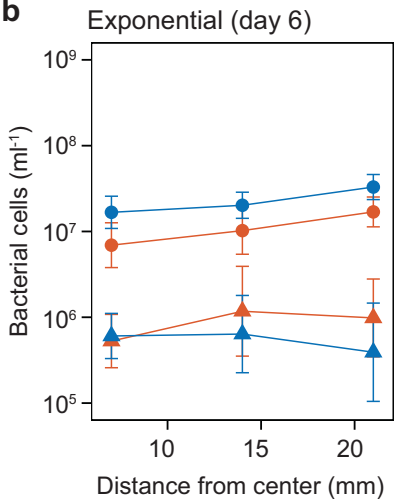

C

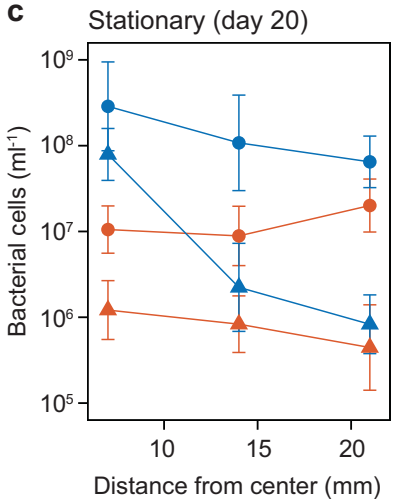

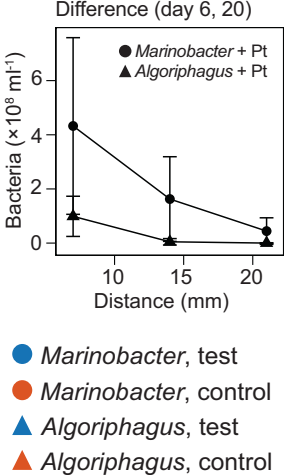

Fig. 5 Measurements of bacterial growth in the porous microplate. a Schematic of a microplate depicting well locations of $P$. tricornutum and two bacterial strains, Marinobacter sp. 3-2 and Algoriphagus sp. ARW1R1. Abundance of the two bacterial strains sampled at (b) exponential and (c) stationary phase co-cultured with $P$. tricornutum (test) or without (control); (inset) difference of the abundance between the timepoints. Error bars, geometric standard deviations [64] of the following number of replicates: $n=5$ (Algoriphagus with $P$. tricornutum, distance $21 \mathrm{~mm}$ ), $n=8$ (Algoriphagus without $P$. tricornutum, distance $21 \mathrm{~mm}$ ), $n=9$ (all others).

co-cultured at different distances from one another (Fig. 5a). The cells were routinely sampled to measure their abundances for three weeks, a period that did not limit algal growth as the surrounding $f / 2$-Si medium is rich with inorganic nutrients. The two bacterial strains were previously isolated from $P$. tricornutum enrichments [16] and are relatively abundant in laboratory cultures where bacterial growth depends on $P$. tricornutum photosynthate [11]. The abundances of the two strains at different time points were compared to one another as a function of the distance away from the center well, which contained either $P$. tricornutum ("test") or f/2-Si medium ("control").

During the initial algal growth phase (day 0-6), we observed a rapid increase of bacterial abundances (Supplementary Fig. S6). Interestingly, Marinobacter was more abundant as distance from the center well increased, regardless of the presence of $P$. tricornutum $(P<0.05$, Fig. 5b). However, Algoriphagus did not exhibit any such difference $(P=0.814$ with algae and $P=0.165$ without). This tendency of higher abundances in the outer wells was also observed for Marinobacter without $P$. tricornutum at the other timepoints in the early growth phase (day $2-8, P<0.05$, Supplementary Fig. S6).

We expect that medium inorganic nutrients were consumed by algal photosynthesis, as shown in our previous experiments, creating a concentration gradient of these inorganic nutrients increasing towards the outer microplate wells (Supplementary Fig. S7). This suggests that Marinobacter sp. 3-2 growth depended on the inorganic nutrients as well as algal DOC at this early growth phase. For the treatment without $P$. tricornutum where we also detected increasing growth towards the outer wells, there was likely also a gradient in inorganic nutrient concentration. This is because the outer wells are adjacent to the medium input outside the microplate, whereas the inner wells are in the center and adjacent to other wells with potentially competing bacteria. Nonetheless, Marinobacter co-cultured with algae were more abundant than without $(P<0.001$, Fig. $5 b)$, suggesting that the bacteria also used some metabolites from $P$. tricornutum for growth and that these metabolites were able to diffuse all the way to the outermost layer.

Results from later in the growth phase (e.g., day 17-20) were drastically different, where the two bacterial strains exhibited distinct responses to the spatial arrangement. On day 20, the abundances of both strains strongly increased with proximity to $P$. tricornutum, indicating that their growth was correlated to algal DOC diffusion $(P<$ 0.001 , Fig. 5c). Algoriphagus appeared to have a greater dependence on $P$. tricornutum exudates than Marinobacter, as measured by a stronger decrease in its abundance with increasing distance from the center (Algoriphagus, 97 fold; Marinobacter, 6 fold). Furthermore, in the outermost well, Marinobacter was 100 times more abundant than Algoriphagus, suggesting that Marinobacter does not require spatial proximity to $P$. tricornutum for its growth. Even noting that initial Marinobacter abundance was ten times greater than Algoriphagus, this still implies that Marinobacter has an ability to utilize $f / 2-\mathrm{Si}$ nutrients for its growth while Algoriphagus does not. Indeed, the growth of Algoriphagus located further away from $P$. tricornutum (in the outer wells) was negligible over the 20 days of incubation period, whereas Marinobacter grew in all the wells (Supplementary Fig. S6). The innermost well was the only location where Algoriphagus grew, suggesting the algal DOC needed by Algoriphagus did not diffuse further, or was fully incorporated by Algoriphagus cells in the innermost well.

\section{Spatial influence on bacterial community development}

Our previous experiment documents the differential response of two bacterial strains grown in monoculture exchanging metabolites with the algal host and potentially with one another. The next step was to test our system with mixed communities and examine how community development is influenced by distance from the alga. By inoculating $P$. tricornutum in the center well and by filling the container with $\mathrm{f} / 2$-Si medium outside (Fig. 6a), we expect that a spatial gradient of increasing inorganic nutrients (higher outside, lower inside) and an opposite gradient of algal DOC (higher inside, lower outside) is generated along the well array, similar to gradients that are hypothesized to exist in the phycosphere at the single cell level. The algal and bacterial cells were incubated in the microplate for a week, a period long enough to elicit population level changes in previous experiments, as discussed above. Bacterial communities were then analyzed by sequencing their $16 \mathrm{~S}$ ribosomal RNA ( $\mathrm{R} R \mathrm{NA}$ ) genes, enabling us to examine how community development is affected by physical location along the two gradients.

For a global analysis, samples were grouped by treatment (with or without $P$. tricornutum in the middle well) and layer number (layer 1 located at the inner and layer 2 at the outer well). Data visualized using principal coordinate analysis ( $\mathrm{PCOA}$ ) showed a notable difference between layer 1 and layer 2 regardless of the treatment $(P<0.001$, Fig. $6 \mathrm{~b}$ inset), suggesting the community structure was influenced by $\mathrm{f} / 2-\mathrm{Si}$ nutrient availability. In both layers, the sample groups were statistically different between treatments $(P<0.05)$, but the structural difference was most visibly obvious in layer 1 compared with layer 2 with a higher proportion 

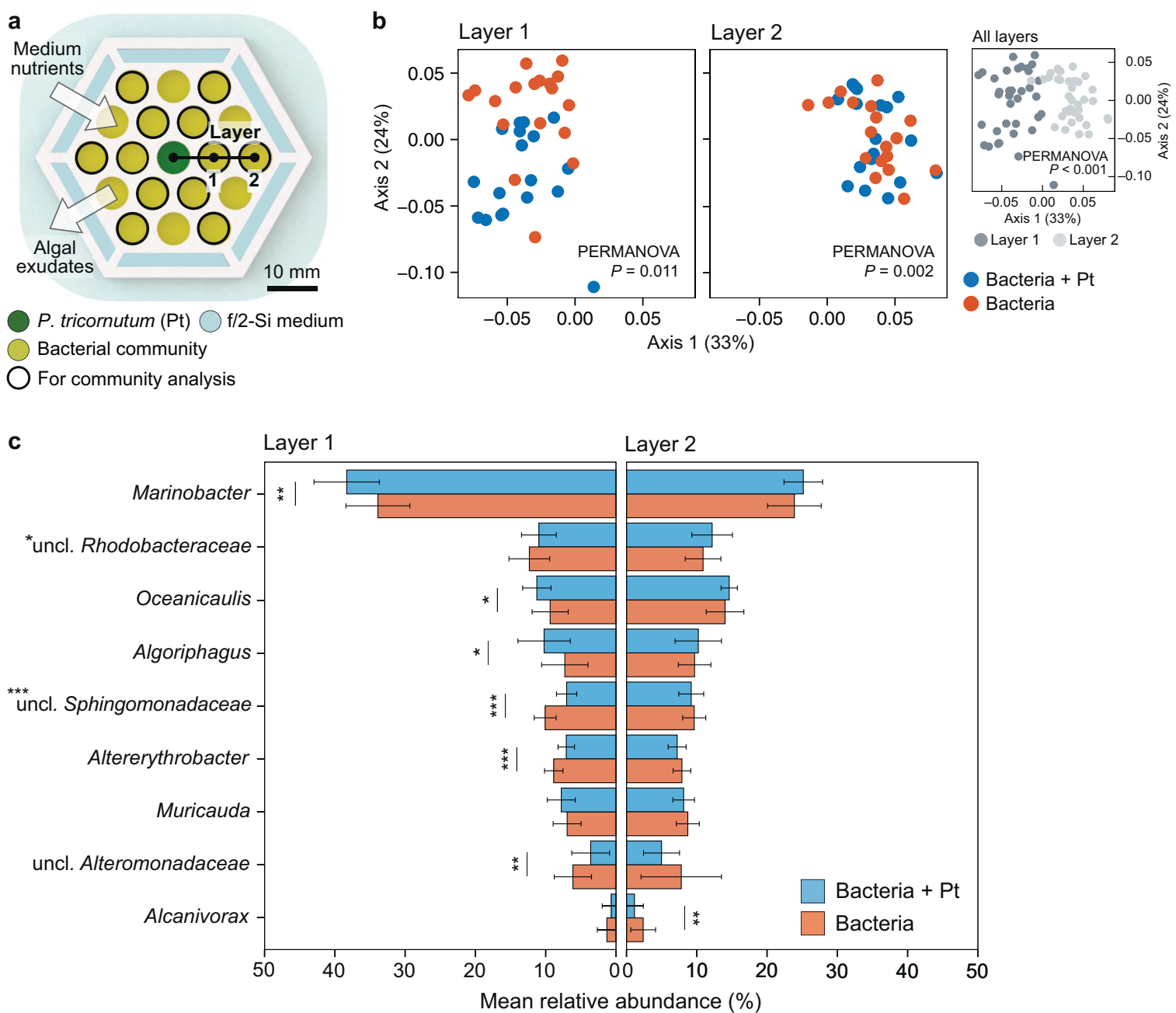

Fig. 6 Bacterial community analysis in the porous microplate. a Schematic of a microplate depicting locations of $P$. tricornutum and associated bacterial communities. b Principal coordinate analysis of the communities using weighted Unifrac metric [46] with $P$-values (PERMANOVA) on structural difference between layer conditions (inset) and the two treatments. c Genus level taxonomic abundance of the communities comparing two treatments incubated for 7 days (means more than $0.5 \%$ displayed). Error bars, standard deviations of 18 replicates. Asterisks next to genera (y-labels), statistically significant interaction term of layer number and treatment (two-way ANOVA). Asterisks next to color bars, statistical significance of differences between two treatments (one-way ANOVA) with following levels: ${ }^{* * *} P<0.001$, ${ }^{* *} P<0.01, *{ }^{*} P<0.05$.

of the variance explained by the principal coordinates (Fig. 6b, Supplementary Fig. S8). This suggests that the community structure was indeed affected by algal photosynthetic activity and exudate production, and to a greater extent for bacterial populations located closest to $P$. tricornutum. Across all 72 community samples, nine ASVs were ubiquitous (found in all samples), assigned to the genera Muricauda, Marinobacter, Oceanicaulis, Altererythrobacter and the families Rhodobacteraceae and Sphingomonadaceae (details provided in Supplementary Fig. S9).

To investigate in greater detail which taxa were influenced by spatial proximity to the algal cells, relative abundances were quantified by merging amplicon sequence variant (ASV) reads at the genus level and compared between well location (inner versus outer) and treatment (with and without algae in the center well). Comparing between treatments (with or without algae), the communities incubated closest to the center well (layer 1) contained more genera that were statistically different in their abundance than in layer 2 (Fig. 6c). In detail, six genera, Marinobacter, Oceanicaulis, Algoriphagus, unclassified Sphingomonadaceae, Altererythrobacter and unclassified Alteromonadaceae, were different in layer 1, whereas only Alcanivorax showed such difference in layer $2(P<0.05)$. We also observed an interaction between treatment and well location with genera Marinobacter, unclassified Rhodobacteraceae, unclassified Sphingomonadaceae, Muricauda and Altererythrobacter, suggesting that they were affected by physical proximity to $P$. tricornutum in the center well and inorganic nutrients on the outside of the microplate $(P<0.05)$. Specifically, the genera Marinobacter, Oceanicaulis, Algoriphagus, and Muricauda exhibited higher relative abundances in cocultures with algae compared to without, suggesting that their response to the algal exudate was stronger than their response to inorganic nutrients. On the other hand, the genera unclassified Rhodobacteraceae, unclassified Sphingomonadaceae, Altererythrobacter and unclassified Alteromonadaceae were relatively less abundant with algae compared to without, indicating they were more strongly influenced by inorganic nutrients, or more likely did not respond to algal exudates as well as the other taxa.

\section{DISCUSSION}

One major unanswered question in the study of algal-bacterial interactions is how the spatial and temporal dynamics of algal exudation and inorganic nutrient uptake contribute to microbial community development around the phycosphere [11, 52]. 
Designing experiments to answer such questions with standard laboratory cultures has been particularly difficult, as no method exists to isolate the contributions of these components. We shed some insight on the problem by using spatial arrays of culture wells with a porous microplate and studied how bacteria grew under different nutritional levels of algal exudates and inorganic compounds. After analyzing bacterial responses both as single isolates and at the mixed community level, we found evidence that certain taxa became more abundant towards algal-derived organic matter, while others responded more strongly to inorganic nutrients away from the algal cells. The latter finding is unexpected, particularly since the initial bacterial communities originated from algal co-cultures where the only source of organic carbon was algal photosynthesis.

Our findings have a major impact on our current understanding of how free-living heterotrophic bacteria respond to the microscale environment around an algal cell. The concept of the phycosphere is based on the idea that organic matter exuded by the algal cell creates a gradient of decreasing concentrations going away from the alga that specific bacteria can take advantage of, and our experimental and modeling data support this well-developed idea. Our experimental and modeling data also suggest that there is a potentially equally important gradient that has thus far been largely ignored: the gradient of inorganic nutrients, which in this case is opposite, increasing in concentration away from the algal cell. It may at first seem odd that inorganic nutrient gradients have not been investigated in the context of heterotrophic bacterial community structure, despite the known ability of bacteria to consume inorganic compounds such as nitrate [51, 53-56], phosphate [53,57] and ammonium [57]. However, heterotrophic bacteria in surface waters are generally known as producers of inorganic nutrients rather than consumers [3], and since algae incorporate more of those inorganic nutrients than heterotrophic bacteria (due to their larger sizes and higher nutrient flux constants), bacteria are not thought to be affected by inorganic nutrient gradients around algal cells. Our data suggest that this assumption is incorrect, and may be particularly critical in high inorganic nutrient ecosystems, such as coastal oceans and engineered algal biofuel ponds designed to maximize the growth of algae [58]. We show that a subset of the bacterial community in these environments can benefit from both inorganic nutrients and organic exudates, suggesting that there are at least 2 different niches in the phycosphere which should support at least 2 bacterial lifestyles. The first niche supports bacteria adapted to incorporate complex algal organic matter, and these organisms only respond to the organic matter gradient and grow best closest to the algal cells and later in the bloom or culture growth cycle, when inorganic nutrients are depleted and algal exudates are more complex (and diffuse slower). This first niche is exemplified by Algoriphagus. The second niche, here represented by Marinobacter, is filled by bacteria that grow using both algal organic matter and inorganic nutrients, and these organisms are likely mutualistic in the early growth phase because there are enough inorganic nutrients for both algae and bacteria (thus no competition for inorganic nutrients). It is likely that these bacteria could become competitive under low inorganic nutrient concentrations, unless they switch their metabolisms from incorporating both organic and inorganic nutrients to incorporating only organic nutrients, a concept that remains untested as of now.

Of further note, our co-culture system illuminates how bacterial responses are governed by the combination of algal growth phase and physical distance in a taxon-specific manner. We observed Algoriphagus exhibiting a greater spatial dependency on $P$. tricornutum than Marinobacter in the isolates experiment, and these responses were confirmed in mixed populations in the community experiment. These experimental observations in the porous microplate enabled us to conceptualize a unique nutrient profile spatially determined by algal growth phase, and we were able to construct a mathematical model of nutrient concentrations around an alga (Fig. 4a), which simultaneously addresses gradients of exudates and inorganic nutrients under different laboratory growth phases. This model allows us to estimate how the algal microbiome might respond to the spatial distribution of the two nutritional factors (algal exudates and inorganic nutrients), because we expect certain bacteria to reproduce at faster rates in locations where more nutrients are accessible. Specifically for the exponential algal growth stage, we expect certain bacteria to exhibit a faster growth rate outside of the phycosphere (i.e. freeliving), because there are still inorganic nutrients available in the bulk medium and smaller molecules exuded from the algal cells can diffuse further away. On the other hand, for algal cultures in stationary phase, other bacteria tend to attach to algal cells or stay within the phycosphere, since inorganic nutrients are depleted throughout the medium and larger molecules diffuse slowly from the surface of the algal cells. Indeed, this scenario explains some experimental observations where the number of attached bacteria increased as algal cultures aged [13, 59].

It should be noted that our proposed system does not address responses of bacteria that physically attach to algae, which is another major contribution to phycosphere interactions [24]. Physical attachment allows bacteria to use high molecular weight carbon compounds that are bound to the surface such as polysaccharides [60], but they have negligible diffusivity in water and in the porous copolymer HEMA-EDMA. To circumvent this limitation, in the single isolates experiment we have selected model organisms that do not attach or rarely attach to $P$. tricornutum even though the organisms were isolated from a "phycosphere enrichment" community $[11,16]$. Nonetheless, the design does not explain whether other algal-attaching bacteria will respond and how they impact host physiology, which is important but remains unanswered.

Notably, by adopting the porous microplate system we constructed a unique environment for algal cells distinct from standard batch cultures, where in the microplate nutrients constantly diffused into the growing culture from outside through the nanoporous copolymer. A supply of fresh nutrients into an algal culture is often found in (semi-) continuous systems; however, our system is also distinct from such methods since algae are not removed from the culture and they accumulate to a high cell density. Indeed, $P$. tricornutum reached abundances significantly higher than previously reported values, including under (semi-) continuous systems [61, 62]. We believe this is the first demonstration that algae can accumulate to such high densities, purely driven by a novel cultivation technique. If appropriately scaled up, this new cultivation approach could lead to more efficient algal biomass production.

Overall, the combination of our experimental culture system and a simple microscale diffusion model successfully explained the temporal and spatial association patterns between algae and different algal-associated bacteria, providing a mechanistic understanding of the distinct impact of inorganic nutrients as well as algal exudates on bacterial growth. We expect this approach can be used in future studies to investigate longstanding questions about community level interactions mediated by metabolite exchange at different spatial scales, including testing hypotheses about bacteria-phycosphere interactions [11, 13, 16], but also more general microbiome interactions in soil, plant, and animal models.

\section{REFERENCES}

1. Amin SA, Parker MS, Armbrust EV. Interactions between diatoms and bacteria. Microbiol Mol Biol Rev. 2012;76:667-84. 
2. Kazamia E, Czesnick H, Nguyen TTV, Croft MT, Sherwood E, Sasso S, et al. Mutualistic interactions between vitamin B12-dependent algae and heterotrophic bacteria exhibit regulation. Environ Microbiol. 2012;14:1466-76.

3. Azam F, Fenchel T, Field J, Gray J, Meyer-Reil L, Thingstad F. The ecological role of water-column microbes in the sea. Mar Ecol Prog Ser. 1983;10:257-63.

4. Amin SA, Green DH, Hart MC, Küpper FC, Sunda WG, Carrano CJ. Photolysis of iron-siderophore chelates promotes bacterial-algal mutualism. Proc Natl Acad Sci. 2009;106:17071-6.

5. Durham BP, Sharma S, Luo H, Smith CB, Amin SA, Bender SJ, et al. Cryptic carbon and sulfur cycling between surface ocean plankton. Proc Natl Acad Sci. 2015;112:453-7.

6. Croft MT, Lawrence AD, Raux-Deery E, Warren MJ, Smith AG. Algae acquire vitamin B12 through a symbiotic relationship with bacteria. Nature. 2005;438:90-3.

7. Cooper MB, Kazamia E, Helliwell KE, Kudahl UJ, Sayer A, Wheeler GL, et al. Crossexchange of B-vitamins underpins a mutualistic interaction between Ostreococcus tauri and Dinoroseobacter shibae. ISME J. 2019;13:334-45.

8. Amin SA, Hmelo LR, van Tol HM, Durham BP, Carlson LT, Heal KR, et al. Interaction and signalling between a cosmopolitan phytoplankton and associated bacteria. Nature. 2015;522:98-101.

9. Behringer G, Ochsenkühn MA, Fei C, Fanning J, Koester JA, Amin SA. Bacterial communities of diatoms display strong conservation across strains and time. Front Microbiol. 2018;9:1-15.

10. Mönnich J, Tebben J, Bergemann J, Case R, Wohlrab S, Harder T. Niche-based assembly of bacterial consortia on the diatom Thalassiosira rotula is stable and reproducible. ISME J. 2020;14:1614-25.

11. Kimbrel JA, Samo TJ, Ward C, Nilson D, Thelen MP, Siccardi A, et al. Host selection and stochastic effects influence bacterial community assembly on the microalgal phycosphere. Algal Res. 2019;40:101489.

12. Krohn-Molt I, Alawi M, Förstner KU, Wiegandt A, Burkhardt L, Indenbirken D, et al. Insights into Microalga and bacteria interactions of selected phycosphere biofilms using metagenomic, transcriptomic, and proteomic approaches. Front Microbiol. 2017;8:1-14.

13. Grossart HP, Levold F, Allgaier M, Simon M, Brinkhoff T. Marine diatom species harbour distinct bacterial communities. Environ Microbiol. 2005;7:860-73.

14. Zehr JP, Weitz JS, Joint I. How microbes survive in the open ocean. Science (80-). 2017:357:646-7.

15. Goldman JC. Conceptual role for microaggregates in pelagic waters. Bull Mar Sci. 1984;35:462-76.

16. Samo TJ, Kimbrel JA, Nilson DJ, Pett-Ridge J, Weber PK, Mayali X. Attachment between heterotrophic bacteria and microalgae influences symbiotic microscale interactions. Environ Microbiol. 2018:20:4385-400.

17. Mayali $X$, Azam F. Algicidal bacteria in the sea and their impact on algal blooms. J Eukaryot Microbiol. 2004;51:139-44.

18. Seymour JR, Simó R, Ahmed T, Stocker R. Chemoattraction to dimethylsulfoniopropionate throughout the marine microbial food web. Science. 2010;329:342-5.

19. Smriga S, Fernandez VI, Mitchell JG, Stocker R. Chemotaxis toward phytoplankton drives organic matter partitioning among marine bacteria. Proc Natl Acad Sci USA. 2016;113:1576-81.

20. Gaines A, Ludovice $M, X u$ J, Zanghi $M$, Meinersmann RJ, Berrang $M$, et al. The dialogue between protozoa and bacteria in a microfluidic device. PLoS One. 2019;14:e0222484.

21. Bell W, Mitchell R. Chemotactic and growth responses of marine bacteria to algal extracellular products. Biol Bull. 1972;143:265-77.

22. Mayali X. NanoSIMS: Microscale quantification of biogeochemical activity with large-scale impacts. Ann Rev Mar Sci. 2020;12:449-67.

23. Stasulli NM, Shank EA. Profiling the metabolic signals involved in chemical communication between microbes using imaging mass spectrometry. FEMS Microbiol Rev. 2016;40:807-13.

24. Seymour JR, Amin SA, Raina J-B, Stocker R. Zooming in on the phycosphere: The ecological interface for phytoplankton-bacteria relationships. Nat Microbiol. 2017;2:17065.

25. Lazier JRN, Mann KH. Turbulence and the diffusive layers around small organisms. Deep Sea Res Part A Oceanogr Res Pap. 1989;36:1721-33.

26. Purcell EM. Life at low Reynolds number. Am J Phys. 1977;45:3-11.

27. Paul C, Mausz MA, Pohnert G. A co-culturing/metabolomics approach to investigate chemically mediated interactions of planktonic organisms reveals influence of bacteria on diatom metabolism. Metabolomics. 2013;9:349-59.

28. Moutinho TJ, Panagides JC, Biggs MB, Medlock GL, Kolling GL, Papin JA. Novel coculture plate enables growth dynamic-based assessment of contact-independent microbial interactions. PLoS One. 2017;12:e0182163.

29. Thøgersen MS, Melchiorsen J, Ingham C, Gram L. A novel microbial culture chamber co-cultivation system to study algal-bacteria interactions using Emiliania huxleyi and Phaeobacter inhibens as model organisms. Front Microbiol. 2018:9:1-12.
30. Ge Z, Girguis PR, Buie CR. Nanoporous microscale microbial incubators. Lab Chip. 2016;16:480-8.

31. Geyer FL, Ueda E, Liebel U, Grau N, Levkin PA. Superhydrophobicsuperhydrophilic micropatterning: Towards genome-on-a-chip cell microarrays. Angew Chem Int Ed. 2011;50:8424-7.

32. Chorazyczewski AM, Huang IS, Abdulla H, Mayali X, Zimba PV. The influence of bacteria on the growth, lipid production, and extracellular metabolite accumulation by Phaeodactylum tricornutum (Bacillariophyceae). J Phycol. 2021:57:931-40.

33. Zobell CE. Studies on marine bacteria. I. The cultural requirements of heterotrophic aerobes. J Mar Res. 1941;4:42-75.

34. Parada $A E$, Needham DM, Fuhrman JA. Every base matters: Assessing small subunit rRNA primers for marine microbiomes with mock communities, time series and global field samples. Environ Microbiol. 2016;18:1403-14.

35. Apprill A, Mcnally S, Parsons R, Weber L. Minor revision to V4 region SSU rRNA 806R gene primer greatly increases detection of SAR11 bacterioplankton. Aquat Micro Ecol. 2015;75:129-37.

36. Illumina. 16S metagenomic sequencing library preparation. Illumina. 2013.

37. Bushnell B BBMap: A Fast, Accurate, Splice-Aware Aligner. 9th Annu. Genomics Energy Environ. Meet. 2014. USDOE Office of Science, Walnut Creek, CA.

38. Callahan BJ, McMurdie PJ, Rosen MJ, Han AW, Johnson AJA, Holmes SP. DADA2: High-resolution sample inference from Illumina amplicon data. Nat Methods. 2016;13:581-3

39. Edgar RC. MUSCLE: Multiple sequence alignment with high accuracy and high throughput. Nucleic Acids Res. 2004;32:1792-7.

40. Price MN, Dehal PS, Arkin AP. Fasttree: Computing large minimum evolution trees with profiles instead of a distance matrix. Mol Biol Evol. 2009;26:1641-50.

41. Murali A, Bhargava A, Wright ES. IDTAXA: A novel approach for accurate taxonomic classification of microbiome sequences. Microbiome. 2018;6:1-14.

42. Quast C, Pruesse E, Yilmaz P, Gerken J, Schweer T, Yarza P, et al. The SILVA ribosomal RNA gene database project: Improved data processing and web-based tools. Nucleic Acids Res. 2013;41:590-6.

43. McMurdie PJ, Holmes S. Phyloseq: An R package for reproducible interactive analysis and graphics of microbiome census data. PLoS One. 2013; 8.

44. Paulson JN, Stine OC, Bravo HC, Pop M. Differential abundance analysis for microbial marker-gene surveys. Nat Methods. 2013;10:1200-2.

45. Anderson MJ. A new method for non-parametric multivariate analysis of variance. Austral Ecol. 2001;26:32-46.

46. Lozupone CA, Hamady M, Kelley ST, Knight R. Quantitative and qualitative $\beta$ diversity measures lead to different insights into factors that structure microbial communities. Appl Environ Microbiol. 2007;73:1576-85.

47. Wickham H, Averick M, Bryan J, Chang W, McGowan L, François R, et al. Welcome to the Tidyverse. J Open Source Softw. 2019;4:1686

48. Oksanen J, Kindt R, Legendre P, Hara B, Henry M, Stevens $H$. The vegan package. Community Ecol Packag. 2007;10:631-7.

49. Faccia PA, Pardini FM, Amalvy Jl. Evaluation of pH-sensitive poly(2-hydroxyethyl methacrylate-co-2-(diisopropylamino)ethyl methacrylate) copolymers as drug delivery systems for potential applications in ophthalmic therapies/ocular delivery of drugs. Express Polym Lett. 2015;9:554-66.

50. Biddanda B, Benner R. Carbon, nitrogen, and carbohydrate fluxes during the production of particulate and dissolved organic matter by marine phytoplankton. Limnol Oceanogr. 1997;42:506-18.

51. Diner RE, Schwenck SM, McCrow JP, Zheng H, Allen AE. Genetic manipulation of competition for nitrate between heterotrophic bacteria and diatoms. Front Microbiol. 2016;7:880.

52. Gonzalez A, King A, Robeson MS, Song S, Shade A, Metcalf JL, et al. Characterizing microbial communities through space and time. Curr Opin Biotechnol. 2012;23:431-6.

53. Øvreås L, Bourne D, Sandaa R, Casamayor E, Benlloch S, Goddard V, et al. Response of bacterial and viral communities to nutrient manipulations in seawater mesocosms. Aquat Micro Ecol. 2003;31:109-21.

54. Allen $A E$, Booth $M G$, Verity PG, Frischer ME. Nitrate uptake by heterotrophic bacteria and the diversity of bacterial nitrate assimilation genes in marine systems. Aquat Micro Ecol. 2005;39:247-55.

55. Wawrik B, Boling WB, van Nostrand JD, Xie J, Zhou J, Bronk DA. Assimilatory nitrate utilization by bacteria on the West Florida Shelf as determined by stable isotope probing and functional microarray analysis. FEMS Microbiol Ecol. 2012;79:400-11.

56. Rodrigues RMNV, Williams PJLB. Inorganic nitrogen assimilation by picoplankton and whole plankton in a coastal ecosystem. Limnol Oceanogr. 2002;47:1608-16.

57. Kirchman DL. The uptake of inorganic nutrients by heterotrophic bacteria. Micro Ecol. 1994;28:255-71.

58. Guillard RRL Culture of Phytoplankton for Feeding Marine Invertebrates. In: Smith WL, Chanley MH (eds). Culture of Marine Invertebrate Animals. 1975. Springer US, Boston, MA, p 29-60. 
59. Riemann L, Steward GF, Azam F. Dynamics of bacterial community composition and activity during a mesocosm diatom bloom. Appl Environ Microbiol. 2000;66:578-87.

60. Azam F, Smith DC. Bacterial influence on the variability in the ocean's biogeochemical state: a mechanistic View. Particle Analysis in Oceanography. 1991. Springer Berlin Heidelberg, Berlin, Heidelberg, pp 213-36.

61. Fabregas J, Patino M, Morales ED, Cordero B, Otero A. Optimal renewal rate and nutrient concentration for the production of the marine microalga Phaeodactylum tricornutum in semicontinuous cultures. Appl Environ Microbiol. 1996;62:266-8.

62. Sharp JH, Underhill PA, Hughes DJ. Interaction (allelopathy) between marine diatoms: Thalassiosira pseudonana and Phaeodactylum tricornutum. J Phycol. 1979;15:353-62.

63. Wong $\mathrm{H}$, Morris S, Radke CJ. Three-dimensional menisci in polygonal capillaries. J Colloid Interface Sci. 1992;148:317-36.

64. Kirkwood TBL. Geometric Means and Measures of dispersion. Biometrics. 1979;35:908-9.

\section{ACKNOWLEDGEMENTS}

We thank T. J. Samo for isolating Marinobacter sp. 3-2, S. Smriga for guidance with culture maintenance, P. Boisvert with SEM imaging, M. Jennings with flow cytometry, S. Kim for advice on statistical analysis. We are grateful to reviewers for their valuable comments. The work was supported by the Department of Energy's Genome Sciences Program grant SCW1039. Work at LLNL was performed under the auspices of the US Department of Energy at Lawrence Livermore National Laboratory under Contract DE-AC52- 07NA27344. H.K. was partly supported by the Kwanjeong Educational Foundation.

\section{AUTHOR CONTRIBUTIONS}

$\mathrm{XM}$ and CRB conceived the study. HK and XM designed the experiment. HK and CAV synthesized porous microplates. JRW isolated nucleic acids, prepared libraries and collected sequencing data. JAK and HK performed bioinformatic analysis. HK developed the mathematical model. HK, JAK, and XM interpreted the data. All authors wrote the paper.

\section{COMPETING INTERESTS}

The authors declare no competing interests.

\section{ADDITIONAL INFORMATION}

Supplementary information The online version contains supplementary material available at https://doi.org/10.1038/s41396-021-01147-x.

Correspondence and requests for materials should be addressed to Xavier Mayali or Cullen R. Buie.

Reprints and permission information is available at http://www.nature.com/ reprints

Publisher's note Springer Nature remains neutral with regard to jurisdictional claims in published maps and institutional affiliations.

(i) Open Access This article is licensed under a Creative Commons Attribution 4.0 International License, which permits use, sharing, adaptation, distribution and reproduction in any medium or format, as long as you give appropriate credit to the original author(s) and the source, provide a link to the Creative Commons license, and indicate if changes were made. The images or other third party material in this article are included in the article's Creative Commons license, unless indicated otherwise in a credit line to the material. If material is not included in the article's Creative Commons license and your intended use is not permitted by statutory regulation or exceeds the permitted use, you will need to obtain permission directly from the copyright holder. To view a copy of this license, visit http://creativecommons. org/licenses/by/4.0/.

(c) The Author(s) 2021 